\title{
COMPARISON OF MICROSCOPIC DRIVERS' PROBABILISTIC LANE-CHANGING MODELS WITH REAL TRAFFIC MICROSCOPIC DATA
}

\begin{abstract}
The difficulties of microscopic-level simulation models to accurately reproduce real traffic phenomena stem not only from the complexity of calibration and validation operations, but also from the structural inadequacies of the sub-models themselves. Both of these drawbacks originate from the scant information available on real phenomena because of the difficulty in gathering accurate field data.

This paper studies the traffic behaviour of individual drivers utilizing vehicle trajectory data extracted from digital images collected from freeways in Iran. These data are used to evaluate the four proposed microscopic traffic models. One of the models is based on the traffic regulations in Iran and the three others are probabilistic models that use a decision factor for calculating the probability of choosing a position on the freeway by a driver. The decision factors for three probabilistic models are increasing speed, decreasing risk of collision, and increasing speed combined with decreasing risk of collision. The models are simulated by a cellular automata simulator and compared with the real data. It is shown that the model based on driving regulations is not valid, but that other models appear useful for predicting the driver's behaviour on freeway segments in Iran during noncongested conditions.
\end{abstract}

\section{KEY WORDS}

simulation, driver behaviour, cellular automata, car following, lane changing

\section{INTRODUCTION}

Traffic simulation has evolved a great deal from its beginnings in the 1950s and 1960s. This, of course, is partly related to the development of computer technology and programming tools. On the other hand, the research in traffic and transportation engineering has also advanced during this 40-year period. Simulation is now an everyday tool for practitioners and researchers in all fields of the profession.

The applications of traffic simulation programs can be classified in several ways. Some basic classifica- tions are the divisions between microscopic, mesoscopic and macroscopic, and between continuous and discrete time approach.

In the following, some of the near-term development trends are discussed. Most of these trends are related to microscopic simulation. One of the oldest and best known cases of the use of simulation in theoretical research is the car-following analysis based on the GM models. In these models a differential equation governs the movement of each vehicle in the platoon under analysis [1]. Car-following, like the intersection analysis, is one of the basic questions of traffic flow theory and simulation, and still under active analysis [2]. Most traffic system simulation applications today are based on the simulation of vehicle-vehicle interactions and are microscopic in nature.

TRANSIMS is an example of still another change in the approach. The traditional traffic flow descriptions are based on continuous speed and distance variables. TRANSIMS, in turn, uses a discrete approach where the road and street network is built from elements that can accommodate only one vehicle at a time unit. In this cellular automata approach, the vehicles move by "jumping" from the present element to a new one [3, 4] according to rules that describe the driver behaviour and maintain the basic laws of physics present in vehicle movements. In cellular automata a basic freeway segment is divided into cells. Individual vehicles in each simulation time step occupy single cells and their movements in these cells describe movement of vehicles on the freeway $[5,6,7]$. Cellular automata are used for modelling pedestrian walking behaviour as a discrete choice model [8], where there are no regulations and no lane to be considered.

Traffic flow simulation rule based approaches, such as HUTSIM and TRANSIMS, are becoming more and more common. In this kind of framework the use of fuzzy logic to describe the human perception can easily be used, and there are several applications of fuzzy car-following models available $[9,10,11]$. 
In most of the studies dealing with traffic behaviour, vehicle movements are defined in two models, car-following and lane-changing $[12,13,14,15]$, which are applied separately. Usually, in car-following models, it is assumed that drivers reach their desired speed in their current lane as they prevent any collision with the vehicle in front. When a driver is not able to reach its desired speed, lane changing to the neighbouring lanes is considered $[16,17,18,19]$. Most of the laneschanging models are based on the assumption that drivers evaluate the current and adjacent lanes and choose the lane with higher average speed. There are also a few models that consider untidiness of traffic flow and lack of drivers' observance to driving lanes [20, 21, 22, 23].

Two kinds of microscopic traffic simulator models, MTS, are studied in this research; separation MTS1 and combination MTS2 models. Time-based simulation software is provided that uses cellular automata to simulate traffic behaviour. This simulation software is capable of micro simulating the drivers' behaviour with the four models illustrated in Figure 1 and described below:

a) Separation model where car-following and lanechanging models are separated, MTS1;

b) Combination model based on decreasing the risk of collision, MTS2A;

C) Combination model based on increasing the speed, MTS2B; and

d) Combination model based on increasing the speed and decreasing the risk of collision, MTS2C.

In MTS1 model, the drivers try to drive in their current driving lane adjusting their speed according to the driving regulations. When speed on the current lane is less than the desired speed of a driver, he will consider the adjacent lanes for lane changing. If the situation on the left lane is better than the current lane, the driver will move to the left lane. Otherwise, he will stay in the current lane. In this way, car following and lane changing is done sequentially.

It has been shown that drivers' behaviour in many countries can not be modelled by the traffic regulations and drivers do not consider driving lanes [24, 25]. The MTS2 models are based on the realities of drivers' behaviour in the freeways of such countries.

In MTS2 models, motional behaviour of drivers is modelled as an optimization process for increasing

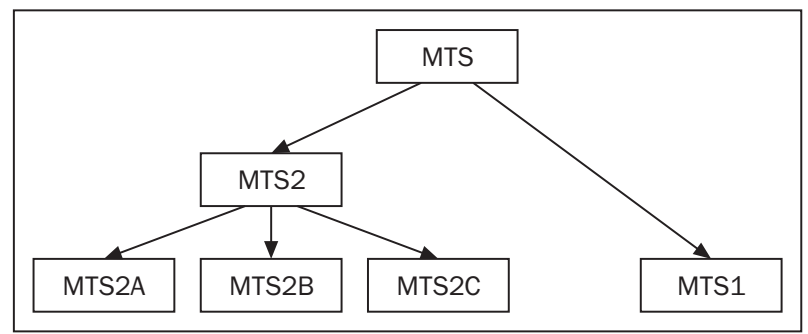

Figure 1 - Relation between the proposed models speed, or decreasing risk of collision, or increasing speed along with decreasing risk of collision. In this way, car-following and lane-changing models are combined as a simultaneous process.

There are many studies that use vehicle trajectories for calibrating their proposed models [26]. In this research an Image processing system is used for collecting vehicles positions in the real world. A freeway in Iran is used as a case study. The data is used for validating the proposed models.

\section{SEPARATION MODEL FORMULATION}

In the separation model MTS1, a freeway with four driving lanes is simulated using the driving regulations in Iran. The freeway is divided into cells. Each cell is as long as the vehicle length, about 5 metres, and as wide as the lane width, about 4 metres.

In car following it is assumed that every driver tries to increase the speed to their desired speed, considering the safe distance to the front vehicle. According to the driving regulations in Iran, for each $10 \mathrm{~km} / \mathrm{h}$ speed, drivers must adjust their distance to the front vehicle about 1.5 times the vehicle length, where the safe distance is calculated as

$d s(I D, t)=\frac{V(I D, t)}{10} *\left(1.5 * L_{I D}\right)$

and where

$d s(I D, t)$ - safe distance between vehicle ID and the front vehicle at time $t$,

$V(I D, t)$ - speed of vehicle ID at time $t$ in kilometre per hour, and

$L_{I D}$ - length of vehicle ID in metres.

Using Eq. 1, maximum safe speed for vehicle ID with distance $d(I D, t)$ from the front vehicle is calculated as

$V_{M S S}(I D, t)=\frac{10 * d(I D, t)}{1.5 * L_{I D}}$

The algorithm that describes drivers' behaviour in MTS1 is depicted in Figure 2. The desired speed is defined for each driver equal to the speed limit. A driver calculates the safe speed in the current lane and compares it with his desired speed. If the safe speed is lower than the desired speed in the current lane, the driver will consider changing to the left lane and calculate the safe speed in that lane. If the safe speed in the left lane is higher than the safe speed in the current lane, the driver will change lanes to the left lane; otherwise he will consider moving into the right lane. If the safe speed in the right lane is higher than the safe speed in the current lane, he will change lanes to the right lane; otherwise, he will remain in the current lane and will adjust his speed to the safe speed.

There is some research dealing with potential collision speeds (PCS) that shows that PCS is a pre- 
dictor of impact speed in a potential chain collision that is dependent on visibility, speed and separation between individual vehicles. The drivers appeared to respond predominantly to their own perceptions and reduced speeds when they faced a risky situation, but not nearly enough to compensate for the risk ahead [27].

\section{COMBINATION MODEL FORMULATION}

The MTS2 model is proposed to model the real drivers' behaviour. The combination model MTS2 is a cellular automata model that combines lane changing and car-following models as a random process for increasing the decision factor. The freeway is divided into cells, where each cell is as long as the vehicle length, about 5 metres, and as wide as the vehicle width, 2 metres. In the MTS2A model, the decision factor is risk, which means that this model is based on a random process for decreasing the crash risk. In the MTS2B model, the decision factor is speed and in the MTS2C model, the decision factor is utility, which is defined as increasing speed along with decreasing the crash risk. The MTS2 models driver behaviour in three phases:

a) determining the feasible cells,

b) calculating the decision factor of the feasible cells,

c) randomly choosing a cell with respect to its decision factor.

Maximum safe speed, risk value, and feasible cells are defined below. The algorithm for the MTS2A model is shown in Figure 3.

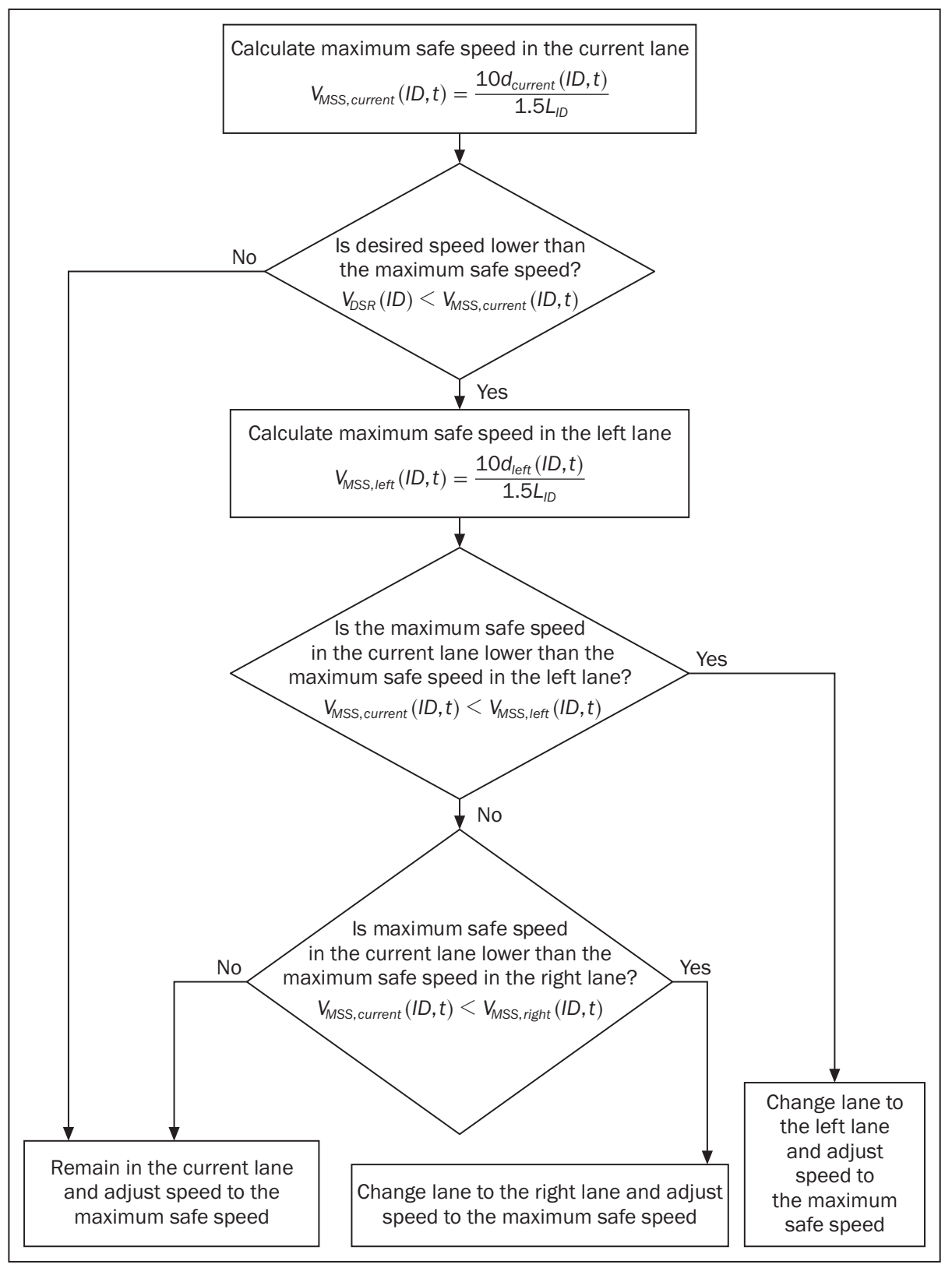

Figure 2 - Algorithm of drivers' behaviour in MTS1 


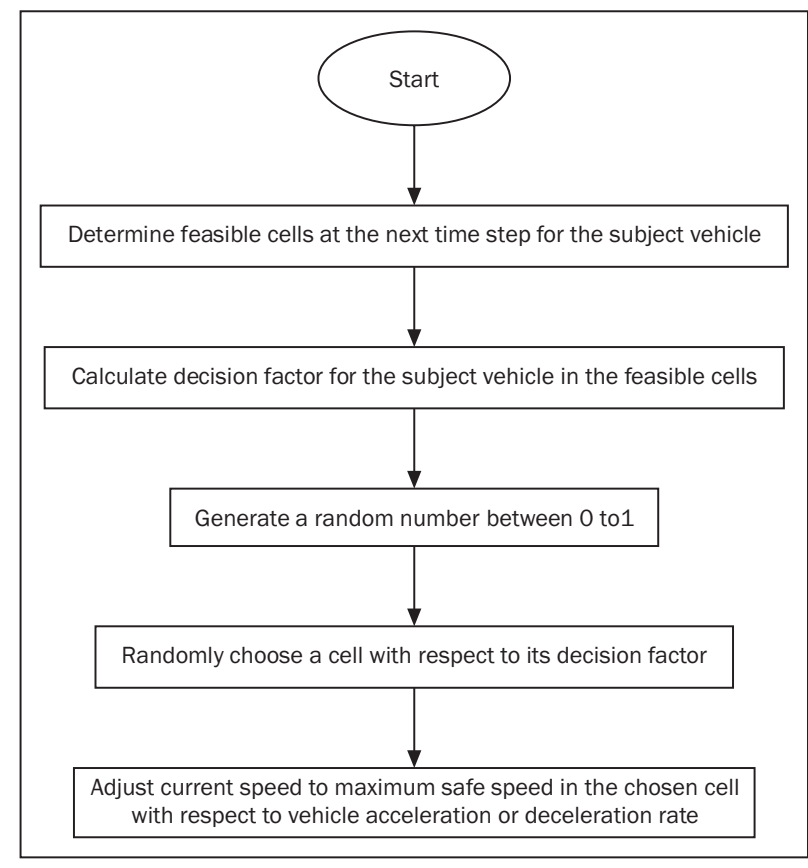

Figure 3 - Applied algorithm in MTS2A model

According to Figure 3, drivers calculate the decision factor for their feasible cells using a probabilistic process that allows for different behaviours in the same situation. Drivers prefer to go to the cells with higher decision factor, but it does not mean that always the cell with the highest decision factor is chosen. Rather, it means that the probability of choosing the cell with the larger decision factor is more than the probability of choosing the cell with the smaller decision factor. The probability of choosing the cell $(X, Y)$ by vehicle ID at time $t$ is calculated from Eq. 3, where the sum of the probabilities of all of the feasible cells equals 1 .

$\operatorname{Pr}(X, Y, I D, t)=\frac{D F(X, Y, I D, t)}{\sum_{i, j} D F(i, j, I D, t)}$

where sum is taken over all feasible cells $(i, j)$ where:

$\operatorname{Pr}(X, Y, I D, t)$ - probability of choosing cell $(X, Y)$ by vehicle ID at time t,

$D F(X, Y, I D, t)$ - decision factor of the cell $(X, Y)$ for vehicle ID at time t.

A random number between 0 and 1 is generated. This number is used for choosing the cells at the next time step. After choosing the cell, the driver must adjust his speed to the maximum safe speed of the chosen cell with respect to deceleration or acceleration of his vehicle. If the current speed of the vehicle is lower than the maximum safe speed, the driver will increase his speed with respect to the acceleration rate of his vehicle. If the current speed of the vehicle is higher than the maximum safe speed, the driver will decrease his speed with respect to the deceleration rate of his vehicle.

\subsection{Maximum safe speed}

Maximum safe speed, $V_{M S S}$, of a cell is the maximum speed that a vehicle can reach in that cell avoiding collision with the front vehicle. Maximum safe speed of a cell is determined by the position and speed of the vehicles in front of vehicle ID.

Thus, the maximum safe speed of vehicle ID for preventing collision with the front vehicles is calculated as [25].

$V_{M S S}(X, Y, I D, t)=-b_{I D} \tau+\sqrt{b_{I D}^{2} \tau^{2}+2 b_{I D}\left(d+\frac{V_{f}^{2}}{2 b_{f}}\right)}$

where $V_{M S S}(X, Y, I D, t)$ is the maximum safe speed in cell $(X, Y)$ for vehicle ID at time $t$. As can be seen, the maximum safe speed in a cell is based on the distance between that cell and its front vehicle $d$, speed of the front vehicle $V_{f}$, reaction time of the drivers, and brake deceleration of the two vehicles $b_{I D}$ and $b_{f}$.

\subsection{Crash risk}

The idea of risk is complex, but conventionally its definition has been consistent, namely, as a measure of probability and impact of adverse effects. Crash risk is calculated by multiplying the impact and probability of crash [29]. In this research, crash risk in a cell for vehicle ID is the probability of collision between vehicle ID and any other vehicle in that cell multiplied by the impact of their collision. Impact is 1 for all of the crashes. As there may be more than one vehicle that can cause a collision in a cell, crash risk of vehicle ID in cell $(X, Y)$ at time $t$ is the sum of the risks created by other vehicles as calculated by

$$
\begin{aligned}
\operatorname{Risk} & (X, Y, I D, t)=\sum_{i} \operatorname{Impact}(X, Y, I D, t) \cdot P(X, Y, i, t)= \\
& =\sum_{i} P(X, Y, i, t)
\end{aligned}
$$

where sum is taken over all $i \neq I D$

where

$\operatorname{Risk}(X, Y, I D, t)$ - crash risk for vehicle ID in cell $(X, Y)$ at time $\mathrm{t}$,

Impact $(X, Y, I D, t)$ - collision impact of vehicle ID in cell $(X, Y)$ at $t$ which is set to 1 and,

$P(X, Y, i, t)$ - presence probability of vehicle i to be in cell $(X, Y)$ at $t$.

An accident occurs when two vehicles occupy the same cell. Therefore, collision probability is the presence probability of two vehicles in the same cell.

Supposing that acceleration is distributed as a normal distribution with average $\mu_{a x}$ and standard deviation $\sigma_{a x}$, the presence probability of vehicle $i$ being at longitudinal position $X$ can be calculated by

$P(i, X, t+\Delta t)=\int_{X-\frac{V_{V}}{2}}^{X+\frac{I_{V}}{2}}$ Normal $_{x}\left(x ; x_{i}(t)+V_{x}(i, t) \cdot \Delta t+\right.$ 


$$
\left.+\frac{1}{2} \mu_{a x} \cdot \Delta t^{2}, \frac{1}{2} \sigma_{a x} \cdot \Delta t^{2}\right) \cdot d x
$$

The position of each cell is determined by the position of the centre of that cell. The lengths of all of the cells are equal to $I_{v}$. Therefore, the lower and upper boundaries of the integral are $X-I_{v} / 2$ to $X+I_{v} / 2$.

In the same way as for longitudinal position, the presence probability of vehicle $i$ being in the lateral position $Y$ can be calculated by

$$
\begin{aligned}
& P(i, Y, t+\Delta t)=\int_{Y-\frac{W_{v}}{2}}^{Y+\frac{W_{v}}{2}} \text { Normal }_{y}\left(y ; y_{i}(t)+V_{y}(i, t) \cdot \Delta t+\right. \\
& \left.+\frac{1}{2} \mu_{a y} \cdot \Delta t^{2}, \frac{1}{2} \sigma_{a y} \cdot \Delta t^{2}\right) \cdot d y
\end{aligned}
$$

Once again the positions of the cells are determined by the position of their centre. The widths of all of the cells are equal to $W_{v}$. Therefore, the lower and upper boundaries of the integral are $Y-W_{v} / 2$ to $Y+W_{v} / 2$.

\subsection{Feasible cells}

Feasible cells for a vehicle are the cells which are options for that vehicle to be in at the next time step considering speed, acceleration, or deceleration of that vehicle. If vehicle ID at time $t$ is in cell $(X(I D, t)$, $Y(I D, t))$ and it is moving at longitudinal speed of $V_{x}(I D, t)$ and lateral speed of $V_{y}(I D, t)$, and acceleration and brake deceleration rates are $a_{I D}$ and $b_{I D}$ respectively, vehicle ID at $t+\Delta t$ will be in the range of feasible cells given by

$$
\begin{gathered}
X(I D, t)+\Delta t^{*} V_{x}(I D, t)-\left(b_{I D}^{*} \Delta t^{2}\right) / 2 \leq X(I D, t+\Delta t) \leq \\
\leq X(I D, t)+\Delta t^{*} V_{x}(I D, t)+\left(a_{I D}^{*} \Delta t^{2}\right) / 2
\end{gathered}
$$

\subsection{Driver utility}

Utility of a driver is an index for measuring how much comfort a driver feels when driving. Drivers prefer to increase their speed and decrease the risk of collision. It is assumed that the utility function is nonlinear and can be calculated as

$U(X, Y, D, t+\Delta t)=\frac{V_{M S S}(X, Y, D, t+\Delta t)^{\alpha} \cdot \rho^{D}}{\operatorname{Risk}(X, Y, D, t+\Delta t)^{\beta}}$

where $\alpha, \beta$, and $\rho$ are coefficients to be determined.
As drivers prefer to move straight forward, the cell that is in front of the vehicle is chosen more often. Therefore, a dummy variable $D$ is defined to include the effect of being in the same lateral position as

$D= \begin{cases}1 & \text { for the cells in the same lateral position } \\ 0 & \text { otherwise }\end{cases}$

Eq. 9 can be written in linear form as follows: $\ln (U(X, Z, I D, t+\Delta t))=\alpha \cdot \ln \left(V_{M S S}(X, Y, I D, t+\Delta t)\right)-$

$-\beta \cdot \ln (\operatorname{Risk}(X, Y, I D, t+\Delta t))+D \ln \rho$

\subsection{Decision factor}

All of the combination models are random models for increasing the decision factor. In the MTS2A model, the decision factor is risk where inverse of risk is used in the model. In the MTS2B model, the decision factor is speed and in the MTS2C model, the decision factor is utility, as summarized in Table 1.

\subsection{Vehicle acceleration and deceleration}

When a vehicle chooses a cell to move to in the next time step, it must adjust its speed to the maximum safe speed in that cell. This cannot occur immediately and must be done according to the vehicle acceleration or deceleration characteristics.

If the current speed of vehicle ID is lower than the maximum safe speed, it can increase its speed based on its acceleration. Thus, the speed of the vehicle in the next time step can be determined using

$V(I D, t+\Delta t)=V(I D, t)+a_{I D} * \Delta t$

If the current speed of vehicle ID is higher than the maximum safe speed, it must decrease its speed based on its deceleration rate. Now the speed of vehicle in the next time step can be determined as

$V(I D, t+\Delta t)=V(I D, t)-b_{I D} \cdot \Delta t$ where:

$b_{I D}-$ brake deceleration of vehicle ID.

\section{DATA COLLECTION}

Two sites, each one covering a basic segment of about 100-metre of the Tehran-Karaj freeway, are

Table 1 - Decision Factor in different models

\begin{tabular}{||c|l|l||}
\hline \hline Model & \multicolumn{1}{|c|}{ Decision factor } & \multicolumn{1}{c|}{ Formulation } \\
\hline \hline MTS2A & Inverse of crash risk & $\operatorname{Risk}(X, Y, I D, t)=\sum_{i} P(X, Y, i, t)$ \\
\hline MTS2B & Maximum safe speed & $V_{M S S}(X, Y, I D, t)=-b_{I D} * \tau+\sqrt{b_{I D}^{2} * \tau^{2}+2 b_{I D} *\left(d+\frac{V_{f}^{2}}{2 b_{f}}\right)}$ \\
\hline MTS2C & Driver utility & $U(X, Y, I D, t)=\frac{V_{M S S}(X, Y, I D, t)^{\alpha} * \rho^{D}}{\operatorname{Risk}(X, Y, I D, t)^{\beta}}$ \\
\hline
\end{tabular}


used for videotaped observation. This freeway connects Tehran to a nearby city Karaj. In the selected segments of the freeway, there are four lanes moving in each direction where each lane is 3.65 meters wide. The camera was installed on a bridge over the freeway giving good view of freeway at two locations. Figures 4 and 5 contain plans of two sites showing camera posi-

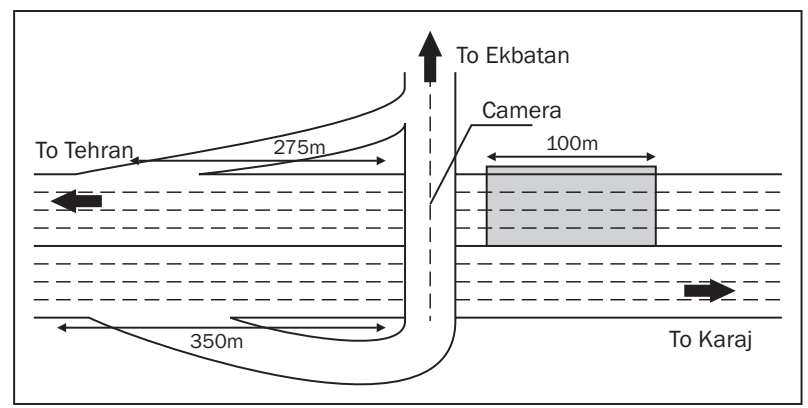

Figure 4 - First site

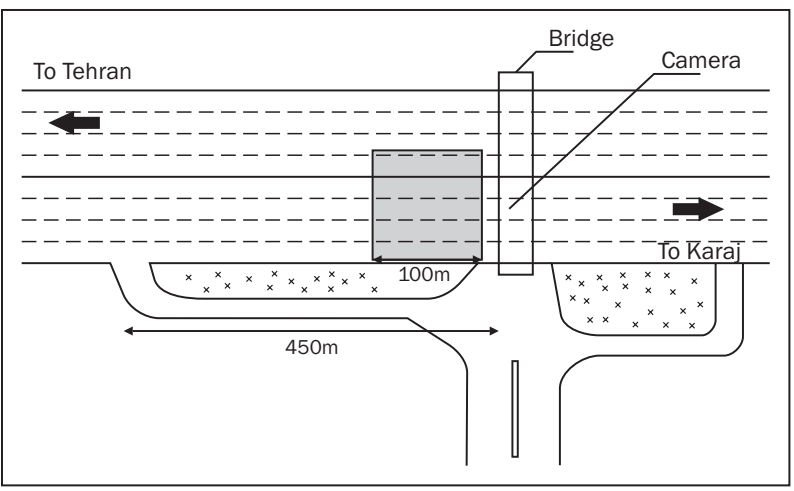

Figure 5 - Second site tion on the bridge and other pertinent distances. The video taped section is indicated by a gray rectangle.

Duration of observation was about 30 minutes at each site. Position, time, and vehicle type passing the segments during the observation in every frame of the film are detected and stored in a table using an image processing system. The image processing program divides the freeway segment into windows for detecting the vehicles. The window size is determined by the ability to produce a complete view of the freeway segment with sufficient resolution and accuracy. Sufficient resolution and accuracy depend on the quality of the image and vehicles image size on the screen. In this case, 20 rows of windows each one containing 12 windows is used for detecting the vehicles. The windows are arranged in horizontal rows, where each row contains 12 windows. Figure 6 shows the windows on a sample image of Tehran-Karaj freeway.

Window positions are determined so that the distances between rows show a specified distance on the freeway, where each window is matched to a specific space on the street [24]. Each window is processed for detecting whether there is a vehicle in it or not. Vehicle positions in each frame are saved in a table in the form of $(I D, X, Y, K, t)$ where

$I D$ - identity number of vehicle,

$X, Y$ - longitudinal and lateral position,

$K$ - vehicle type according to its size (for truck or bus $K=1$, for private car $K=0$ ),

$t$ - time of observance.

Using the table of vehicle positions, the microscopic traffic characteristics of the vehicles are de-

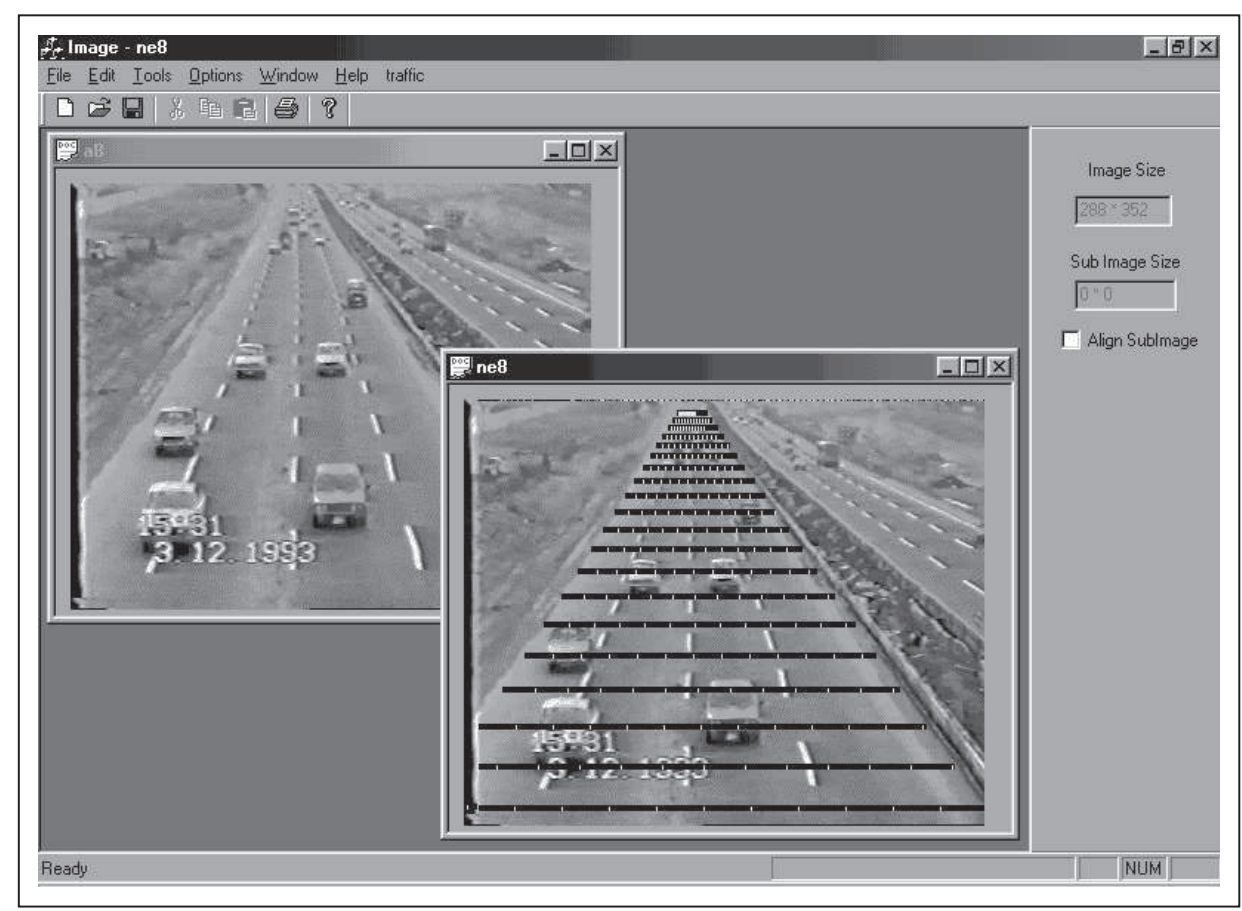

Figure 6 - Windows on a sample image of Tehran-Karaj freeway 
termined. Macroscopic parameters such as average speed are calculated by averaging the speed of individual vehicles.

The first site was observed from 15:30 to 16:00 where 1,392 vehicles were detected. The average speed was about $83 \mathrm{~km} / \mathrm{h}$. The second site was observed from $15: 30$ to $16: 00$ where 1,397 vehicles were detected. The average speed was about $82 \mathrm{~km} / \mathrm{h}$.

\section{MODEL CALIBRATION}

For calibrating the maximum safe speed $V_{\text {MSS }}$ in Eq. 4 , the deceleration rate is set to $5 \mathrm{~m} / \mathrm{s}^{2}, L$ is set to 5 , and reaction time is set to 2 seconds for all the vehicles [30]. With these values Eq. 4 can become

$V_{M S S}(X, Y, I D, t)=-10+\sqrt{50+10 d+V_{f}^{2}}$

Using the above equation, the maximum safe speed in cells can be calculated according to the distance to the nearest front vehicle $d$, and speed of the nearest front vehicle $V_{f}$.

The acceleration rate of vehicles $a_{x}(i, t)$ in Eq. 12 must be calculated in order to calibrate the presence probability of vehicle $i$ in longitudinal position $X$. Acceleration $a_{x}(i, t)$ is calculated for different vehicles $i$ at different times $t$. A sample of $a_{x}$ is prepared which is used for calculating the frequency of different values of $a_{x}$ in the sample. Thus, the estimate of the distribution function of $a_{x}$ is given as

$$
\min \sum_{a_{x}}\left(\text { frequency }\left(a_{x}\right)-\operatorname{Normal}_{x}\left(a_{x} ; \mu_{a x}, \sigma_{a x}\right)\right)^{2}
$$

for all observed $a_{x}$

where:

frequency $\left(a_{x}\right)$ - frequency of observing longitudinal acceleration $a_{x}$ in the sample data,

$\operatorname{Normal}_{x}\left(a_{x} ; \mu_{a x}, \sigma_{a x}\right)$ - value of $a_{x}$ in normal distribution with average $\mu_{a x}$ and standard deviation $\sigma_{a x}$.

The frequency of $a_{x}$ in the observed sample can be best fitted as a normal distribution with average $\mu_{a x}=0$ and standard deviation $\sigma_{a x}=9$. Normality of $a_{x}$ is tested using the Kolmogorov-Smirnov test and Chi-square test, which give the distribution function as normal at $95 \%$ level of confidence. Considering $I_{v}=5$, Eq. 6 is calibrated as

$$
P(i, X, t+\Delta t)=\int_{x-2.5}^{x+2.5} \text { Normal }_{x}\left(x ; x_{i}(t)+V_{x}(i, t) . \Delta t\right.
$$

$$
\text { 4.5. } \left.\Delta t^{2}\right) . d x
$$

for the same method is used to calculate the presence probability of vehicle $i$ at lateral position $Y, a_{y}(i, t)$ for different vehicles $i$ at different times $t$. A sample of $a_{y}$ is chosen and the frequency of different values of $a_{y}$ in the sample are calculated. It turns out that the frequency of $a_{y}$ can be fitted as a normal distribution with average $\mu_{a y}=0$ and standard deviation $\sigma_{a y}=3$. Normality of $a_{y}$ is tested using Kolmogorov-Smirnov test and Chi-square test. Here, the distribution function is normal at $99 \%$ level of confidence. Considering $W_{v}=2$, Eq. 7 is calibrated as

$$
\begin{gathered}
P(i, Y, t+\Delta t)=\int_{Y-1}^{Y+1} \operatorname{Normal}_{y}\left(y ; y_{i}(t)+V_{y}(i, t) \cdot \Delta t,\right. \\
\text { 1.5. } \left.\Delta t^{2}\right) \cdot d y
\end{gathered}
$$

For calibrating the utility function in Eq. 11, it is assumed that feasible cells are limited to the 3 front cells, as in the sample data. Then the frequency percentage of vehiclesthatchoosethefrontcell, $U(X+1, Y, I D, t+\Delta t)$, left cell, $U(X+1, Y-1, I D, t+\Delta t)$, and right cell, $U(X+1, Y+1, I D, t+\Delta t)$ in the next time step $t+\Delta t$, is calculated using the sample data as $0.97,0.017$, and 0.013 , respectively. Considering the utility of the feasible cells to be the decision factor in Eq. 3 and probability to be equal to frequency percentage of the cells, Eq. 11, can be calibrated with the coefficients $\alpha$, $\beta$, and $\rho$ determined using a linear regression method. Thus

$$
\begin{aligned}
& \ln (U(X, Y, I D, t))=16.4 . \ln \left(V_{M S S}(X, Y, I D, t)\right)- \\
& \quad-4.5 . \ln (\operatorname{Risk}(X, Y, I D, t))+D \ln \left(7.89 * 10^{13}\right)
\end{aligned}
$$

where all of the variables are defined in Eq. 11 and $R^{2}=0.94$ for Eq. 18 .

\section{AN EXAMPLE OF DRIVERS' BEHAVIOUR}

Figure 7 gives an example of moving vehicles in a segment of freeway where vehicles are moving from left to right. The subject vehicle is shown in a black cell by number 1; other vehicles are shown in gray cells. Each cell is a 5 by 2 metre rectangle on the freeway surface. The considered section is a 4-lane freeway, each lane about 4 metres in width. Thus, the width of the freeway is separated into 8 cells.

In this example the speed limit is $120 \mathrm{~km} / \mathrm{h}$. Speeds of vehicles 3 and 4 are $108 \mathrm{~km} / \mathrm{h}$ and speeds of vehicles 1 and 2 are $72 \mathrm{~km} / \mathrm{h}$. Assuming the time step to be 0.2 second, vehicles 3 and 4 will move about 6 metres and vehicles 1 and 2 will move about 4 metres in the next time step. Since the freeway cells are 5 metres long, the simulation moves all of the vehicles one cell forward and adds 1 metre to the distance travelled by vehicles 3 and 4 , but subtracts 1 metre from the distance travelled by vehicles 1 and 2 in the next time step.

In the MTS1 model, the driver calculates the safe speed in the current lane in the first phase. Since there are 3 cells between the considered vehicle and the front vehicle, there is a 15-metre distance from the front vehicle. Using Eq. 2, the maximum safe speed of the considered vehicle in the current lane, left lane and right lanes are $72 \mathrm{~km} / \mathrm{h}, 48 \mathrm{~km} / \mathrm{h}$, and $120 \mathrm{~km} / \mathrm{h}$, 


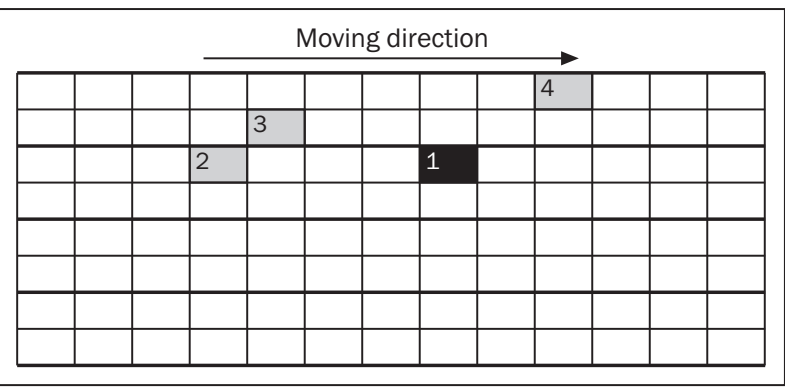

Figure 7 - Considered vehicle and other vehicles on the street

respectively. Using the MTS1 model the considered driver will change lanes to the right cell.

The maximum safe speed over 3 feasible cells of the considered vehicle in the MTS2 models are calculated using Eq. 14. The maximum safe speed for the front cell is $16 \mathrm{~m} / \mathrm{s}$, right cell $25 \mathrm{~m} / \mathrm{s}$, and left cell $33 \mathrm{~m} / \mathrm{s}$.

The presence probabilities of vehicles for the left, front, and right cells with respect to the considered vehicle are calculated using Eq. 3 and shown in Table 2. For the cells that are influenced by more than one vehicle, the sum of presence probabilities is chosen as the true presence probability.

Table 2 - Presence probability of vehicles in the feasible cells

\begin{tabular}{|l||c|c|c||c||}
\hline \multicolumn{1}{|c||}{ ID } & 2 & 3 & 4 & sum \\
\hline \hline Left & $1.96 \mathrm{E}-25$ & $8.35 \mathrm{E}-17$ & $1.77 \mathrm{E}-06$ & $1.77 \mathrm{E}-06$ \\
\hline Straight & $3.72 \mathrm{E}-24$ & $4.41 \mathrm{E}-18$ & $1.06 \mathrm{E}-11$ & $1.06 \mathrm{E}-11$ \\
\hline Right & $1.96 \mathrm{E}-25$ & $2.65 \mathrm{E}-23$ & $0.00 \mathrm{E}+00$ & $2.65 \mathrm{E}-23$ \\
\hline \hline
\end{tabular}

Risk values of feasible cells are shown in Figure 8.

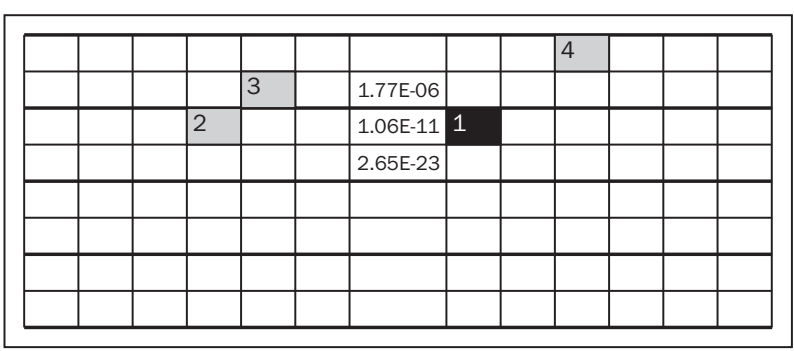

Figure 8 - Risk values created for the considered vehicle

Vehicle 1 considers the utility of its feasible cells and chooses a cell accordingly. The utilities of feasible cells are calculated using Eq. 18 and are shown in Figure 9.

$$
\begin{aligned}
& \ln \left(U_{l}\right)=16.4 \cdot \ln (25)-4.5 \cdot \ln \left(1.77 * 10^{-6}\right)= \\
& \quad=112 \\
& \ln \left(U_{s}\right)=16.4 . \ln (16)-4.5 \cdot \ln \left(1.06 * 10^{-11}\right)+ \\
& \quad+\ln \left(7.89 * 10^{13}\right)=191 \\
& \ln \left(U_{r}\right)=16.4 \cdot \ln (33)-4.5 \cdot \ln \left(2.6 * 10^{-23}\right)= \\
& \quad=291
\end{aligned}
$$

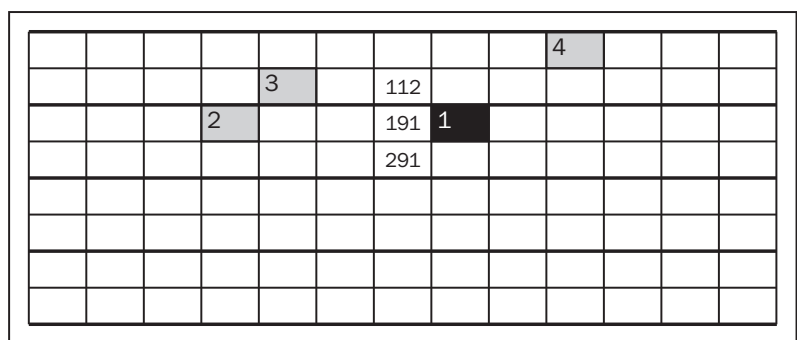

Figure 9 - Utility of feasible cells for the considered vehicle

Probability of choosing feasible cells in the MTS2 models is calculated using Eq. 3 and shown in Table 3.

Table 3 - probability of choosing the cells in MTS2 models

\begin{tabular}{||l|c|c|c||}
\hline & & MTS2B & MTS2C \\
\hline \hline $\operatorname{Pr}$ (left) & 0.00 & 0.34 & 0.19 \\
\hline $\operatorname{Pr}$ (front) & 0.00 & 0.22 & 0.32 \\
\hline $\operatorname{Pr}$ (right) & 1.00 & 0.44 & 0.49 \\
\hline
\end{tabular}

A random number is selected between 0 and 1 . If the random number is less than $\operatorname{Pr}($ left), the left cell will be selected. If the random number is greater than $\operatorname{Pr}($ left) and less than $\operatorname{Pr}($ left $)+\operatorname{Pr}$ (front), the front cell will be selected. If the random number is greater than $\operatorname{Pr}($ left $)+\operatorname{Pr}$ (front), the right cell will be selected. In the above example, let us suppose the random number is 0.25 . Then, in MTS2A the driver will choose the right cell, in MTS2B the left cell, and in MTS2C the front cell.

In the MTS2A model, the maximum safe speed of the chosen cell is $33 \mathrm{~m} / \mathrm{s}$. If the speed of vehicle 1 is $72 \mathrm{~km} / \mathrm{h}$ or $20 \mathrm{~m} / \mathrm{s}$, vehicle 1 can increase its speed. Assuming the acceleration rate to be $1.2 \mathrm{~m} / \mathrm{s}^{2}$ and time step to be 0.2 second, vehicle 1 will increase its speed about $0.2 * 1.2=0.24 \mathrm{~m} / \mathrm{s}$. In this way, vehicle 1 will go into the right cell and will increase its speed to $20.24 \mathrm{~m} / \mathrm{s}$ or $72.864 \mathrm{~km} / \mathrm{h}$ in the next time step.

In the MTS2B model, maximum safe speed of the chosen cell is $25 \mathrm{~m} / \mathrm{s}$, so vehicle 1 will increase its speed by about $0.24 \mathrm{~m} / \mathrm{s}$. Thus, vehicle 1 will go to the left cell and will increase its speed to $20.24 \mathrm{~m} / \mathrm{s}$ or $72.864 \mathrm{~km} / \mathrm{h}$ in the next time step.

In the MTS2C model, maximum safe speed of the chosen cell is $16 \mathrm{~m} / \mathrm{s}$, so, assuming its deceleration rate to be $5 \mathrm{~m} / \mathrm{s}^{2}$ and the time step to be 0.2 second, vehicle 1 will decrease its speed about $0.2 * 5=1 \mathrm{~m} / \mathrm{s}$. Thus, vehicle 1 will go to the front cell and will decrease its speed to $19 \mathrm{~m} / \mathrm{s}$ or $68.4 \mathrm{~km} / \mathrm{h}$ in the next time step.

\section{PREPARED MICROSCOPIC TRAFFIC SIMULATION SOFTWARE}

Time-based simulation software was prepared for simulating the movement behaviour of vehicles 
using the proposed models [24]. In the simulation software, the freeway is divided into cells, each cell being almost equal to the length of a private car of 5 metres and width of 2 metres. In each time step, each vehicle occupies a cell and the movement of the vehicles is described by their movement in the cells. Vehicles are created in the time and location where they have been seen for the first time in real data. Only the first position of a vehicle in the simulation is the same as in the real data and the positions in the next time steps are calculated using the proposed models.

\section{VALIDATION OF THE PROPOSED MODEL}

The duration of the observations was about half an hour at each of the two sites. Data collected from the first site were used for calibrating the models and those from the second site for validating the models. Model validation is done both at the microscopic and macroscopic levels. At the macroscopic level, the average speed and the number of lane changes in the simulation and real world are compared and at the microscopic level the longitudinal and the lateral position of the vehicles are compared.

The duration of the observation at the second site is divided into 6 intervals, each one about 5 minutes. The simulation is performed for each of the intervals individually. The vehicles are created in the time and position where they were seen during the first time step in the real world.

There are different methods for validating the microscopic traffic models [31]. In this research, the paired observation test is used for validating the proposed model. Test statistics of the paired observation test is calculated by the equation below:

$$
t_{p o}=\frac{\bar{d}}{S_{d} / \sqrt{n}}
$$

where

$$
\begin{aligned}
t_{p o}- & \text { test statistics, } \\
\bar{d}- & \text { average of differences between real and } \\
& \text { simulation pairs, } \\
S_{d}- & \text { standard deviation of differences between } \\
& \text { real and simulation pairs, } \\
n- & \text { number of pairs. }
\end{aligned}
$$

If $\left|t_{p o}\right|>t_{\alpha / 2, n-1}$, validation of the model is rejected at the $1-\alpha \%$ level of confidence; otherwise, there is no reason to consider the model invalid [32].

\subsection{Macroscopic validation}

All of the four models have been evaluated for simulating the average speed and number of lane changes in the gathered data of the real traffic. A paired observation test is used and $t_{p o}$ is calculated for each of the models as shown in Table 4.

As can be seen, the MTS1 model, which was based on the traffic regulations in Iran, cannot simulate drivers' behaviour and it is rejected. Other models are accepted at the $95 \%$ level of confidence.

\subsection{Microscopic validations}

The root mean square error for the longitudinal position, $t$ seconds after initial position was used as a quantitative parameter for validating the models. For calculating the difference between longitudinal and lateral position of vehicles in the real world and simulation t seconds after initial position, RMSSEIX and RMSSEIY have been used, where RMSSEIX and RMSSEIY are calculated from

$R M S S E I X=\sqrt{\frac{\sum_{I D=F}^{N+F-1}\left(X R\left(t_{\text {in }}(I D)+t\right)-X S\left(t_{\text {in }}(I D)+t\right)\right)^{2}}{N}}$

and where

RMSSEIX - root mean sum of square error $t$ second after initial position,

$N$ - number of detected vehicles,

$F$ - index number of the first detected vehicle,

$X R\left(t_{i n}(I D)+t\right)$ - longitudinal position of vehicle ID in the real world $t$ second after being detected for the first time, and

$X S\left(t_{i n}(I D)+t\right)$ - longitudinal position of vehicle ID in the simulation $t$ second after it is created.

As another quantitative parameter, root mean square error for the longitudinal position for all of the time steps was used for the model validation. The difference between longitudinal position of vehicles in the real world and simulation relies on RMSSETX, which is calculated by

$R M S S E T X=\sqrt{\frac{\sum_{I D=F}^{N+F-1} \sum_{t(I D)=t_{\text {in }}(I D)+1}^{t_{\text {out }}(I D)}(X R(t(I D))-X S(t(I D)))^{2}}{\sum_{I D=F}^{N+F-1}\left(t_{\text {out }}(I D)-t_{\text {in }}(I D)\right)}}$

Table 4 - Macroscopic evaluation results

\begin{tabular}{||c|c|c|c|c||}
\hline \hline MTS2C & MTS2B & MTS2A & MTS1 & Model \\
\hline \hline 0.36 & 1.83 & 2.07 & 56.33 & $t_{p o}$ for average speed \\
\hline 1.69 & 1.15 & 1.15 & 56 & $t_{p o}$ for the number of lane changes \\
\hline accepted & accepted & accepted & rejected & evaluation result \\
\hline
\end{tabular}


where:

RMSSETX - root mean sum of square error for all of the time steps,

$N$ - number of detected vehicles,

$F$ - index number of the first detected vehicle, $X R\left(t_{\text {in }}(I D)+t\right)$ - longitudinal position of vehicle ID in the real world at $t$,

$X S\left(t_{\text {in }}(I D)+t\right)$ - longitudinal position of vehicle ID in the simulation at $\mathrm{t}$,

$t_{\text {out }}(I D)$ - last time that the vehicle ID is seen, and

$t_{\text {in }}(I D)$ - first time that the vehicle ID is detected. In the same way, RMSETY is defined for lateral position of vehicles.

The results of the evaluation are shown in Table 5. As can be seen, all of the parameters related to the MTS2C model are better than for the other models. It can be deduced that it is better to simulate driver's behaviour in Iran by the MTS2C model.

Table 5 - Microscopic evaluation results

\begin{tabular}{||c|c|c|r|c||}
\hline \hline MTS2C & MTS2B & MTS2A & \multicolumn{1}{c|}{ MTS1 } & Parameter/Model \\
\hline \hline 0.47 & 0.57 & 0.60 & 1.10 & RMSSEIX \\
\hline 0.11 & 0.12 & 0.17 & 0.60 & RMSSEIY \\
\hline 1.8 & 2.4 & 4.10 & 123.00 & RMSSETX \\
\hline 0.67 & 0.82 & 1.16 & 23.40 & RMSSETY \\
\hline \hline
\end{tabular}

\section{CONCLUSION}

A cellular model is proposed for simulating the driving behaviour on freeways of Iran. Two kinds of microscopic traffic simulator models are studied in this research, namely, separation and combination models. The separation model is based on the driving regulations in Iran. Combination models are based on a non-stochastic process for maximizing the decision factor. Crash risk, maximum safe speed, and driver utility are used as decision factors in these models. Proposed models are micro simulated using prepared microscopic simulator software. It is shown that the separation model is not valid. However, combination models are valid for describing driving behaviour on freeway basic segments in Iran. Thus, combination models can be used in traffic simulator software for simulating drivers' behaviour in Iran.

This study deals with the basic freeway segments, where drivers' different destinations for exiting or entering the freeway or continuing straight ahead do not affect their movement behaviour. For future studies the destination factor could be added to the optimization process. In this way, the proposed model could be used not only for basic freeway segments, but also on other parts of the road network. In this research, the drivers' behaviour is modelled under non-congested situations, where there are empty cells around the subject vehicle. For future studies, it is advisable to consider traffic dynamics under congested situations.

\section{LITERATURE}

[1] Gerlough, D., and Huber, M. Traffic flow theory. A monograph. TRB Special Report 165. Washington, D.C. (1975)

[2] McDonald, M., Brackstone, M., and Sultan, B., Instrumented vehicle studies of traffic flow models. Proceedings of the Third International Symposium on Highway Capacity, Volume 2, Ryysgaard, R., ed., pp. 755-774. Copenhagen: Transportation Research Board and Danish Road Directorate, (1998)

[3] Nagel, K., and Schleicher, A. Microscopic traffic modelling on parallel high performance computers. Parallel Computing, 20, 125-146, (1994)

[4] Brilon, W., and Wu, N., Evaluation of cellular automata for traffic flow simulation on freeways and urban streets. Tagungsband zum Ergebnis-Workshop: Verkehr und Mobität, Stadt Region Land, Heft 66, pp. 111-117. Aachen: Rheinisch-Westfälische Technische Hochschule Aachen, (1998)

[5] Denos C. Gazis, Herman, R., Rothery, R., Nonlinear Follow-The-Leader Models of Traffic Flow, Operations Research, Vol. 9, No. 4, pp. 545-567, (1961)

[6] Heng, W., and Meyer, E., and Lee, J., and Chuen, F., Characterizing Lane-Choice Behaviour to Build Rules As Part of Lane-Based Traffic Micro-Simulation Hierarchy, Transportation Research Board (CD-ROM), $81^{\text {st }}$ Annual Meeting, (2002)

[7] Sultan, B., The Lane Changing Process: Data Analysis \& Modeling behaviour, Transportation Engineering and Control, pp. 202-207, (2001)

[8] Shahab Uddin, M., and Ardekani, S., An Observation Study of Lane Changing on Basic Freeway Segment, Transportation Research Board (CD-ROM), $81^{\text {st }}$ Annual Meeting, (2002)

[9] Kikuchi, S., and Chakroborty, P., Car-following model based on fuzzy inference system. Transportation Research Record 1365, Transportation Research Board, Washington, D.C., 82-91, (1992)

[10] Rekersbrink, A., Mikroskopische Verkehrssimulation mit Hilfe der Fuzzy-logic. Strassenverkehrstechnik 2/95, 68-74, (1995)

[11] Wu, J., McDonald, M., and Brackstone, M., A fuzzy logic microscopic simulation model for interurban ATT assessment, Proceedings of $10^{\text {th }}$ European simulation symposium (ESS'98), pp. 347-354, (1998)

[12] Kazi Iftekhar, A., Modeling drivers' acceleration and lane changing behaviour, Massachusetts Institute of Technology, (1999)

[13] Gipps, P.G., A behavioral car-following model for computer simulation, Transportation Research B 15: 105111, (1981)

[14] Hidas, P., "A car-following model for urban traffic simulation", Traffic Engineering and Control, vol. 39, pp. 300-305, 1998

[15] Kita, E., Tamaki, T., \& Shimizu, H., Cellular automata simulation of traffic flow through a road with a construction zone, Transactions of the Essex Institute, http://library.witpress.com/pages/Paperlnfo. asp?PaperlD=16769, (2008). 
[16] Nagel K. and Schreckenberg, M., A cellular automaton model for freeway traffic, J. Physique I 2, 2221 (1992)

[17] Esser, J. and Neubert, L. and Wahle, J. and Schreckenberg, M., Microscopic Online Simulations of Urban Traffic Using Cellular Automata, Transportation and Traffic Theory, pp. 535-554, (1999)

[18] Barlovic, R., Esser, J., Froese, K., Online Traffic Simulation with Cellular Automata, http://www.traffic.uniduisburg.de, (2005)

[19] Antonini, G., Bierlaire, M. and Weber, M., Discrete Choice Models of Pedestrian Walking Behaviour, Transportation Research Part B, (CD-ROM), (2006)

[20] Gunay, b., An investigation of lane utilization on Turkish highways, Proceedings of civil engineers, Transport 157, pp. 43-49, (2004)

[21] Gunay, b., Woodward, D., Lateral position of traffic negotiating horizontal bends, Proceedings of civil engineers, Transport 160, pp. 1-11, (2007)

[22] Gunay, b., rationality of a non lane based car following theory, Proceedings of civil engineers, Transport 162, pp. 27-37, (2009)

[23] Gunay, b., Car following theory with lateral discomfort, Transportation Research Part B 41, pp. 722-735, (2007)

[24] Sadat Hoseini, S.,M., Vaziri, M., Using Image processing for Microsimulation of Freeways in Developing
Countries, Transportation Research Board, TRB, (CDROM), (2006)

[25] Sadat Hoseini, S., M., Vaziri, M., Modeling Drivers' Behaviour as a crash risk Reduction Process, PROMETTraffic \& Transportation, Vol. 20, pp 139-146, Zagreb, Croatia, (2008)

[26] Hoogendoorn, S., Ossen, S., Gorte, B., Inter-Driver Differences in Car Following: a Vehicle Trajectory Based Study, Transportation Research Board, TRB, (CD-ROM), (2006)

[27] MacCarley, C Arthur, Ackles, Christopher, Watts, Tabber, Highway Traffic Response to Dynamic Fog Warning and Speed Advisory Messages, Transportation Research Record: Journal of the Transportation Research Board, pp. 95-104, (2006)

[28] Ming-Chin Tsai, Chien-Chih Su, Scenario analysis of freight vehicle accident risks in Taiwan, Accident analysis \& prevention, (2004)

[29] Risk definition,www.wikipedia.com, (accessed 2011)

[30] AASHTO, Geometric Design of Highways and Streets, p. 40, (2004)

[31] Brockfeld, E., Kuhne. R., Wagner, P., Calibration and Validation of Microscopic Models of Traffic Flow, Transportation Research Board, TRB, (2005)

[32] Chung, C., Simulation modeling hand book, CRC PRESS, pp 8.11-8.14, (2003) 